\title{
Design of RF filters with high selectivity on monolithic ceramic blocks
}

\author{
Roman Semernya ${ }^{1, *}$, Artem Vilenskiy ${ }^{1}$, and Sergey Chernyshev ${ }^{1}$ \\ ${ }^{1}$ BMSTU, Electronics and laser technology Department, 105005, Moscow, Russia
}

\begin{abstract}
The paper presents the results of the development of quasielliptic filters on monolithic ceramic blocks. A circuit analysis of equivalent filter circuits is carried out using the coupling matrix method. A novel topology of a monolithic filter is being studied with the aim of obtaining increased frequency-selective properties due to the implementation of the attenuation poles. High cross-coupling values on ceramic monoliths with large inner metalized holes were obtained. The engineering method of adjusting the frequency position of the attenuation poles by trimming capacitive stubs is realized. Based on the performed analysis, as well as electromagnetic modeling, a monolithic ceramic filter was manufactured. The measurements results of the S-parameters for obtained models are presented.
\end{abstract}

\section{Introduction}

Microwave filters are an integral part of electronic communication, navigation and radar systems, performing frequency selection of signals against existing interferences and noise. In particular, microwave filters are able to improve signal to noise ratio in receiving devices, as well as the parameters of electromagnetic compatibility by reducing spurious radiation of transmitting antennas of radio electronic systems.

Due to the rapid development of electronic systems, the requirements for frequencyselective devices are constantly increasing, in particular, the requirements for the shape of frequency response, the position of the additional attenuation poles, the level of insertion loss, etc. At the same time, high requirements for electrical characteristics are certainly accompanied by toughening requirements for the overall dimensions, operational and cost (economic) parameters of the system.

In the frequency bands of navigation data transmission systems, in particular, such as GPS or GLONASS (L-band), it is often possible to fulfill the above requirements using microwave filters based on metalized monolithic ceramic blocks (MCB) (see Fig. 1) [1-5].

As can be seen from Fig. 1, the filters on the MCB are rectangular metalized ceramic blocks with through holes that form the structure of coupled co-directional coaxial resonators, the electromagnetic coupling between which is determined by ceramic parameters, mutual arrangement and size of the metalized holes, and the geometry of face topology. The shaping of the filter face topology is usually based on screen printing or laser engraving technology $[4,5]$.

\footnotetext{
${ }^{*}$ Corresponding author: semernyare@gmail.com
} 


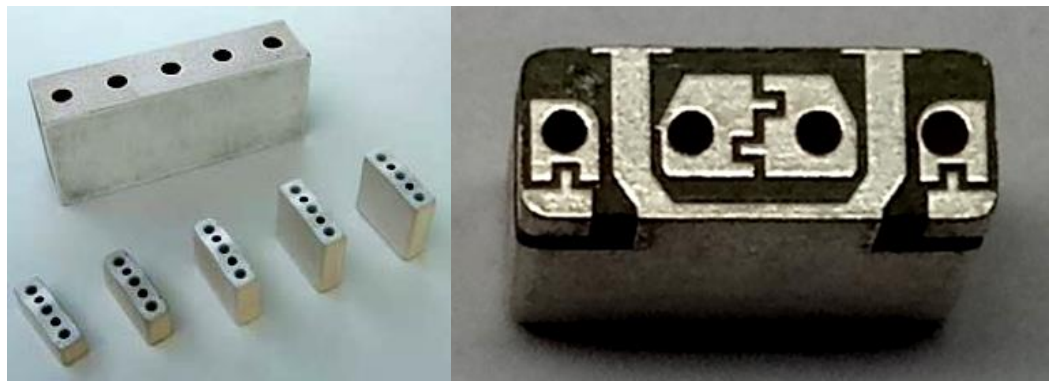

Fig. 1. The view of metalized ceramic blocks and classical topology of the 4th order filter with two attenuation poles.

By choosing ceramics of MCBs with high values of relative permittivity $(20,40$, and 90 ), as well as with a low dielectric loss tangent (usually in the range from 0.0001 to 0.001 ), it is possible to achieve a reduction in filter dimensions, while maintaining a low level of insertion loss by compared with filters on microstrip lines (MSL).

However, despite the advantages, it is very difficult to implement filters on the MCBs with controlled asymmetry of the frequency response due to the inability to independently control and adjust the frequency position of the attenuation poles. The above mentioned difficulty is due to the rigidly set geometric parameters of the MCB design, the sequential arrangement of the resonators in the ceramic block, and the relative dielectric constant of ceramics.

An additional feature of the filter development on MCBs is the limited choice of geometric parameters, type of ceramic and resonator cross sections in the retail sale, which determines the relevance of the development and analysis of new topologies that allow implementing the required types of frequency response on existing MCBs.

In this paper, we consider the application of the recursive method for coupling matrix synthesizing [6], as well as the electromagnetic modeling of coupled coaxial resonators in the MCBs to obtain the face topology of a quasi-elliptic band pass filter (QEF) [7, 8] with a controlled and adjustable attenuation pole position, which significantly increases variety of implemented frequency response forms. The proposed new topology was tested using an example of a fourth-order filter with a relative passband of $10 \%$ with two attenuation poles on an MCB with a relative permittivity of 40 .

\section{Coupling matrix calculation}

On the microwave frequencies it is convenient to use the recursive technique for the coupling matrix synthesis [6] to analyze the structural description of QEF, as well as the design of electrical equivalent circuits for bandpass filters with many cross-couplings. The use of a recursive technique allows calculating the elements of the coupling matrix for the required shape of the QEF frequency response for $N$ iterations, where $N$ is the filter order, without resorting to optimization algorithms, which greatly simplifies the development of the QEF. The key steps of the method are briefly described below.

To specify the initial conditions for the synthesis of the coupling matrix, such as the order of the filter $N$, the number of attenuation poles $N_{z}$, as well as their frequencies $\omega_{n}$, which determine the desired type of frequency response, it is convenient to use the following representation for the $S$-parameters of the filter [6]:

$$
S_{21}^{2}(\omega)=\frac{1}{1+\varepsilon^{2} C_{N}^{2}(\omega)}, C_{N}(\omega)=\cosh \left[\sum_{n=1}^{N} \cosh ^{-1}\left(x_{n}\right)\right], x_{n}=\frac{\omega-1 / \omega_{n}}{1-\omega / \omega_{n}},
$$


where $\omega$ is the circular frequency; $\varepsilon$ - ripple coefficient in the passband; $C_{N}(\omega)-$ filtering function for a band-pass filter with an arbitrary arrangement of attenuation poles. The form of the required frequency response form is shown in Fig. 2.

The main idea of the method is the representation of the QEF scattering parameters in the form of rational fractions:

$$
S_{11}(s)=\frac{F(s) / \varepsilon_{R}}{E(s)}, S_{21}(s)=\frac{P(s) / \varepsilon}{E(s)}
$$

where $E(s), F(s), P(s)$ are the polynomials of the Nth order with coefficients $e_{0}, e_{1}, \ldots, e_{N}, f_{0}, f_{1}, \ldots, f_{N}, p_{0}, p_{1}, \ldots, p_{N}$ determined as a result of the recursive procedure [6-8], $\varepsilon_{R}$ и $\varepsilon$ are the normalizing coefficients, which set the highest polynomials coefficients to unity. For convenience, subsequently, the $S$-parameters are converted to a system of $Y$-parameters.

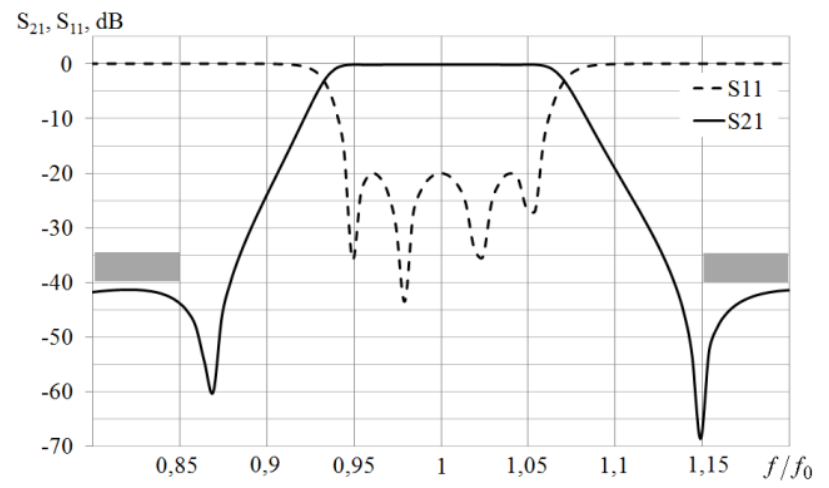

Fig. 2. The frequency response for $4 \mathrm{p} 2 \mathrm{z}$ QEF (4 poles 2 zeros) with required attenuation levels.

Further, using the equivalent prototype filter circuit with the generalized coupling structure shown in Fig. 3 and applying the Kirchhoff' s formula for circuit currents, we can express the internal elements of the prototype filter, such as the coupling coefficients $M_{i j}$ and the reactive elements of the resonator detuning $j B_{i}$ (or self couplings) through $Y$ parameters, previously defined:

$$
y_{21}(s)=[j \overline{\mathbf{M}}+s \mathbf{I}]_{N 1}^{-1}, \quad y_{22}(s)=[j \overline{\mathbf{M}}+s \mathbf{I}]_{N N}^{-1},
$$

where $\overline{\mathbf{M}}$ is matrix of QEF coupling coefficients normalized to the fractional bandwidth $F B W ; \overline{\mathbf{M}}=\mathbf{M} / F B W ; \mathbf{I}$ is the identity matrix. A further calculation of the coupling matrix is based on the Gram-Schmidt orthogonalization procedure and is not further described in detail [6].

It should be mentioned that, as shown in [6], the obtained coupling matrix is not the only solution; therefore, from the point of view of searching for the simplest structural realizations of a given form of frequency response, it is of great interest to find additional solutions. For this, the rotation operator $\mathrm{R}$ is applied to the coupling matrix, which does not change the eigenvalues of the matrix, however, with the correct selection of rotation angles, it allows to zero out some of the elements of the matrix M. The type of transformation is as follows [6]:

$$
\mathbf{M}_{r}^{\prime}=\mathbf{R}_{r} \cdot \mathbf{M}_{r-1} \cdot \mathbf{R}_{r}^{T}
$$




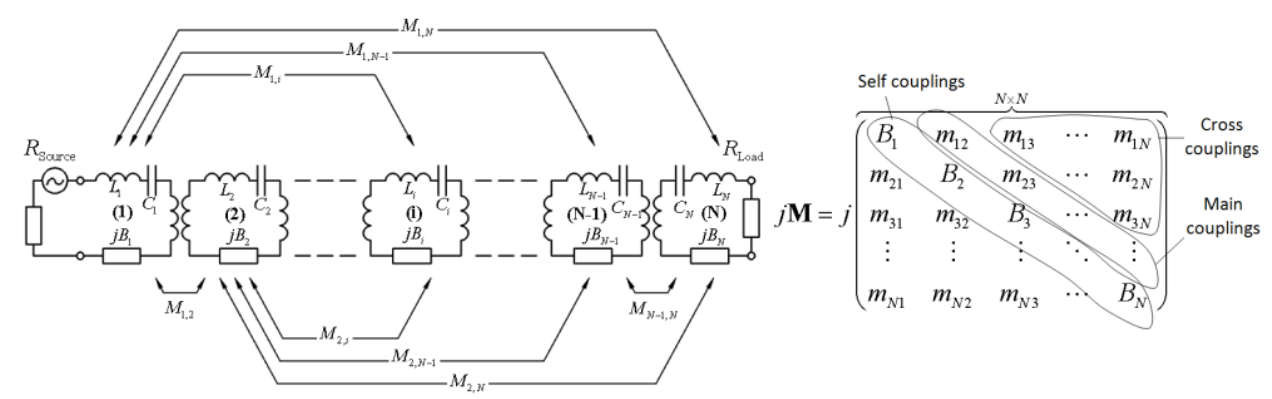

Fig. 3. A QEF bandpass filter prototype circuit that implements an asymmetric frequency response with an arbitrary position of the attenuation poles (left), a general view of the coupling matrix (right).

\section{QEF on MCB analysis}

Using the above algorithm, the coupling matrix of the filter on MCB with the classical topology [3] was analyzed and calculated, and its equivalent circuit was developed (Fig. 4). The parameters of the equivalent circuit are electric lengths $\theta_{\mathrm{r} 1} \ldots \theta_{\mathrm{r} 4}$ and wave impedances $Z_{\mathrm{r} 1} \ldots Z_{\mathrm{r} 4}$ of coaxial resonators, coupling elements of electric $(\mathbf{E})$ and magnetic $(\mathbf{H})$ type, input and output coupling capacitors $C_{S 1}, C_{4 L}$, as well as shortening or tuning capacitors $C_{1} \ldots C_{4}$.

\begin{tabular}{|c|c|c|c|c|}
\hline (a) & 1 & 2 & 3 & 4 \\
\hline 1 & 0 & 0,0889 & 0 & 0,0097 \\
\hline 2 & 0,0889 & 0 & $-0,0739$ & 0 \\
\hline 3 & 0 & $-0,0739$ & 0 & 0,0889 \\
\hline 4 & 0,0097 & 0 & 0,0889 & 0 \\
\hline
\end{tabular}

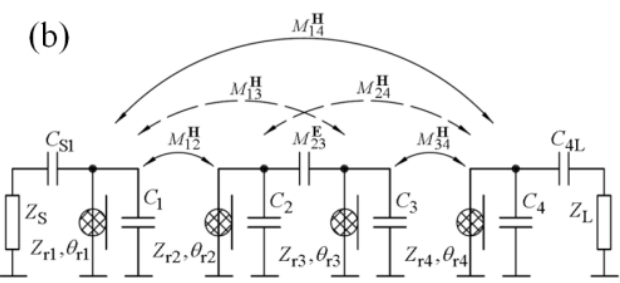

Fig. 4. The coupling matrix of QEF on MCB with classical topology (a) and its equivalent circuit (b).

As can be seen from the obtained coupling matrix and equivalent circuit, in order to obtain two attenuation poles near the passband (normalized passband frequencies are $\pm j 2.55)$ in the classical topology, it is required to provide magnetic coupling between the first and fourth resonators. The cross-couplings indicated by the dashed line are negligible and make an insignificant contribution to the shape of the frequency response, so at this stage they are equal to zero.

As will be shown later using electromagnetic modeling, magnetic cross-couplings have a small range of changes and settings for existing MCBs available on the market, which is a significant disadvantage of this design, limiting the possible frequency-selective properties of QEF with the selected topology.

Figure 5 shows the dependences of the cross-coupling values of the output resonators in $\mathrm{MCB}$ versus the distance between the resonators and the holes diameters given by electromagnetic modeling in HFSS.

A commercially available ceramic block with a relative dielectric constant of 40 , cross section of 10x4 mm, a cavity length of $8.2 \mathrm{~mm}$, a distance between resonators of $2 \mathrm{~mm}$ and a diameter of resonators of $0.6 \mathrm{~mm}$ was chosen.

As can be seen from Fig. 5, for the selected parameters of the MCB, the required value of cross-coupling between the first and fourth resonators is not achieved, which does not allow realizing the frequency response of a given shape with the required attenuation levels. 
However, as can be seen from Fig. 5c, the use of MCB with edge holes of reduced diameters (0.35-0.4 mm) (see Fig. 6) can provide cross-coupling coefficient higher on $0.3 \%$ compared to holes of $0.6 \mathrm{~mm}$. Also, based on the tuning practice, it is possible to slightly increase the magnetic coupling between the output resonators by introducing cutouts on the shorted side of the MCB, which leads to an increase in the equivalent inductance of the resonator and, accordingly, the mutual magnetic coupling between the resonators.

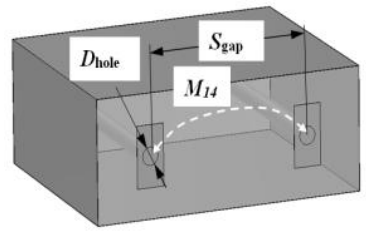

a)

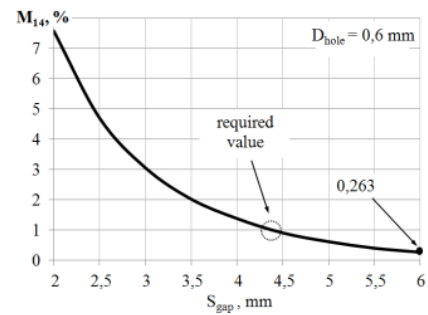

b)

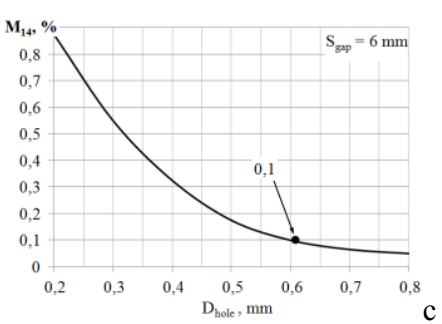

)

Fig. 5. Electromagnetic model of external resonators in HFSS (a), the cross coupling coefficient $v s$ distance between the resonators (b) and diameters of the holes (c).

However, the above-described methods of increasing the coupling coefficients lead to a decrease in the quality factor of the resonators and an increase the level of insertion loss in the passband, which is undesirable.
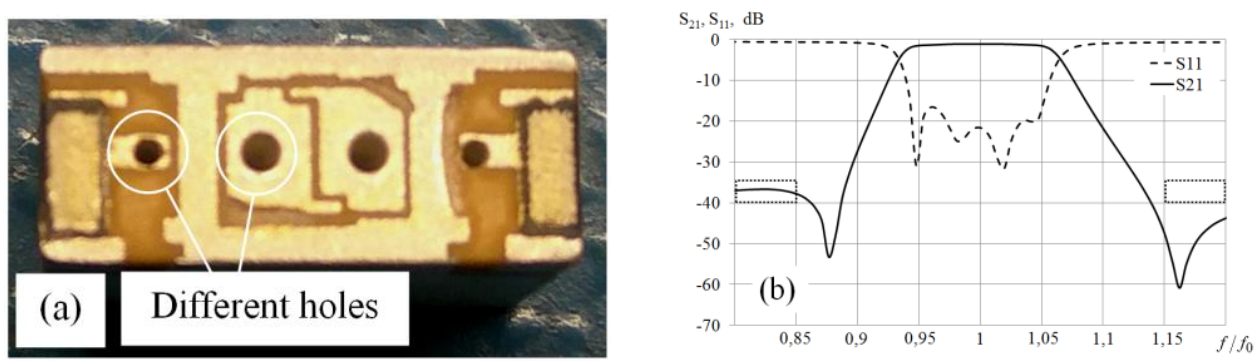

Fig. 6. QEF on MCB prototype with the classical topology (a) and its measured $S$-parameters (b)

As an alternative method of providing the required level of cross-coupling between output resonators, it is proposed to use an equivalent circuit with dual electromagnetic couplings compared to the classical circuit. This will make it possible to realize cross coupling of electrical type, the adjustment of which is often easier to perform with topological elements of face metallization than magnetic coupling. The coupling matrix, as well as the developed equivalent circuit of the proposed QEF are shown in Fig. 7.

\begin{tabular}{|c|c|c|c|c|}
\hline (a) & 1 & 2 & 3 & 4 \\
\hline 1 & 0 & $-0,0889$ & 0 & $-0,0097)$ \\
\hline 2 & $-0,0889$ & 0 & 0,0739 & 0 \\
\hline 3 & 0 & 0,0739 & 0 & $-0,0889$ \\
\hline 4 & 0,0097 & 0 & $-0,0889$ & 0 \\
\hline
\end{tabular}

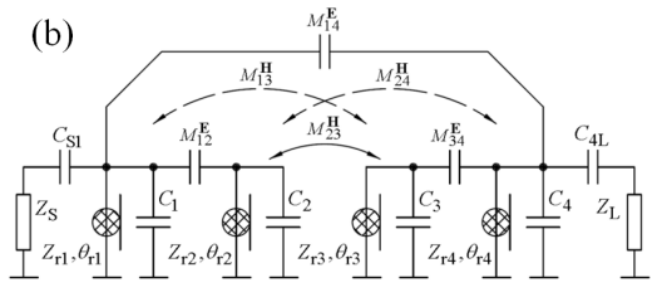

Fig. 7. Coupling matrix of the proposed QEF on MCB (a) and its equivalent circuit (b) 
Further, applying electromagnetic modeling, a face topology was developed corresponding to the equivalent circuit and coupling matrix shown in Fig. 7. The QEF 3D electromagnetic model with a new topology, as well as the dependence of the coupling coefficient between output resonators on the capacitive gap between the resonators are shown in Fig. 8.
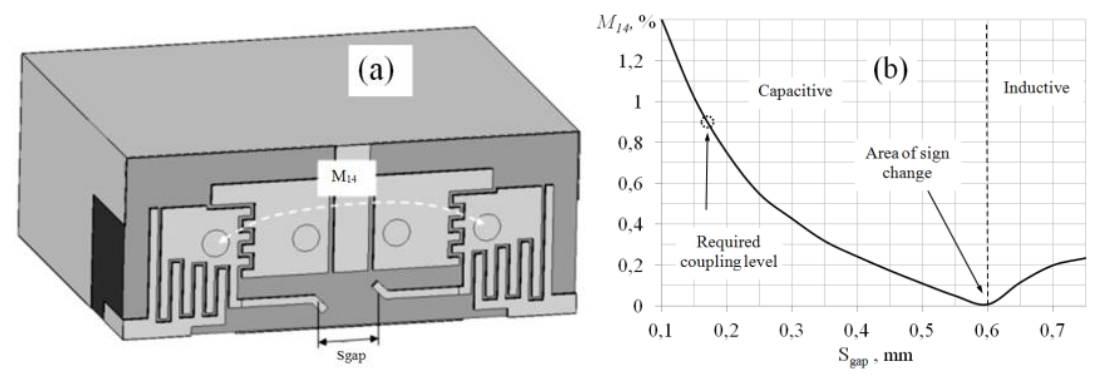

Fig. 8. HFSS 3D electromagnetic model of proposed QEF on MCB (a), dependence of the cross coupling coefficient on the distance between capacitive stubs (b).

As can be seen from Fig. 8, the proposed faced topology parameters make it possible to obtain the required level of cross-coupling, which sets the necessary frequency position of the attenuation poles. Note that as the gap between the stubs of output resonators $S_{\text {pes }}$ increases to $0.6 \mathrm{~mm}$, the coupling decreases to zero and then begins to grow again. This effect is associated with the conversion of the electric type of coupling into magnetic, which is inherent in coupled co-aligned resonators with different phase velocities of an even and odd mode of excitation.

Based on the analysis of electrical circuits, coupling matrices, as well as electromagnetic modeling, a fourth-order QEF was fabricated on MCB with a relative dielectric constant of 40 with electrical coupling between output resonators. The measurement results of the $S$-parameters, as well as the view of face topology are shown in Fig. 9.
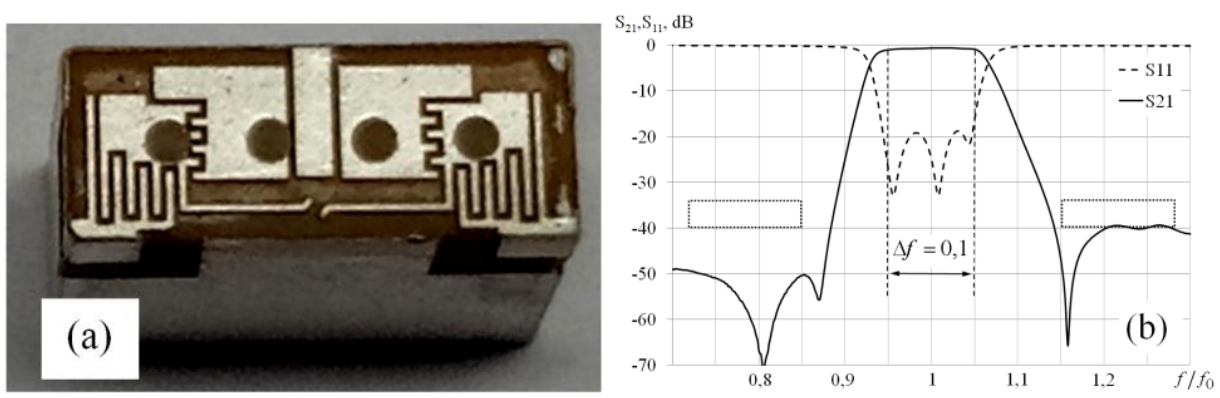

Fig. 9. Proposed QEF on MCB with novel topology (a), measured scattering parameters (b)

Cross coupling tuning in the given prototype can be done by increasing capacitive gap between stubs, thereby moving the attenuation poles away from the passband. Thus, the adjustment of the slope of the frequency response in the attenuation region is regulated without decreasing of the quality factor, and, therefore, does not worsen the loss in the passband.

It should be noted that there are additional attenuation poles and their asymmetric arrangement relative to the passband, especially in a wide frequency band (see Fig. 10). As a more accurate analysis of the filter coupling matrix shows, the appearance of additional poles and the asymmetry of the frequency response are associated with the presence of capacitive coupling between the first and third and, respectively, second and fourth 
resonators $M_{13}^{\mathbf{E}}=M_{24}^{\mathbf{E}}$, as well as the magnetic coupling between the regular microstrip lines $M_{S L}^{\mathbf{H}}$.

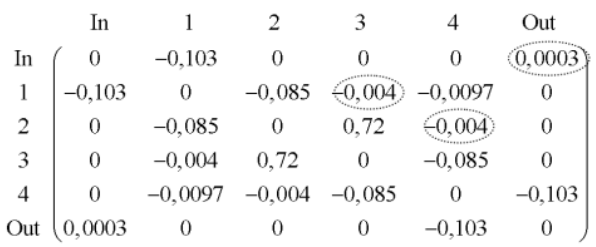

(a)

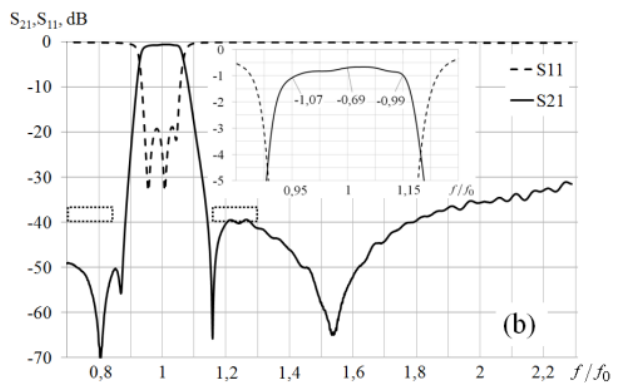

Fig. 10. Refined coupling matrix of the proposed QEF on MCB (a), measured $S$-parameters in a wide frequency band (b).

It should also be mentioned that the obtained frequency response fully satisfy the technical specifications shown in Fig. 2. The cross-coupling between output resonators (the strongest among the cross couplings) makes the greatest contribution to the level of signal attenuation in the given rejection bands; therefore, the initial condition for the absence of additional cross-coupling can be considered acceptable. Total losses are less than 1,07 dB in a $10 \%$ frequency band.

\section{Conclusion}

The method of analysis and calculation of coupling matrices for QEF is considered. An analysis of the QEF on the MCBs with the classical type of topology is carried out, an equivalent circuit is designed. As a result of the circuit analysis, a new face topology for the implementation of the fourth order QEF on MCBs with two attenuation poles, the frequency position of which can be adjusted in a wide frequency band, is presented. This advantage of the proposed topology in comparison with the classical one allows using ceramic blocks widespread on the market for the implementation of frequency response with increased frequency-selective properties.

The electromagnetic modeling of filter coupling coefficients on the MCB was carried out, on the basis of which a QEF prototype was made and an experimental study of its $S$ parameters was carried out.

The proposed geometry of the QEF topology on MCB is fully satisfied the given requirements, and also allows tuning the frequency position of the attenuation poles, which is an undoubted advantage of this topology.

\section{References}

1. A.Iu Beliakov et al., Band-pass ceramic filter with coplanar coupling element, Vestnik Novgorodskogo gosudarstvennogo universiteta im. Jaroslava Mudrogo, №. 7 (98), pp. 71-73 (2016)

2. E.V. Petrov, V.V. Popov, A.Iu Beliakov, Small-size ceramic bandpass filter with wide suppression frequency band, Vestnik Novgorodskogo gosudarstvennogo universiteta im. Jaroslava Mudrogo, v. 1, №. 75, pp. 26-29 (2013)

3. A.Iu Beliakov et al., Development of microwave band-pass filters with arbitrary frequency response, Vestnik Novgorodskogo gosudarstvennogo universiteta im. Jaroslava Mudrogo, №. 8 (91), pp. 45-51 (2015) 
4. Wang X., Wu K. L., Yin W. Y., A novel surface-mounted monoblock dielectric filter, IEEE Transactions on Components, Packaging and Manufacturing Technology, v. 4, №. 11, pp. 1822-1827 (2014)

5. V.A. Kozlov et al., Manufacturing technique coaxial ceramic resonators for microwave devices, Fizika i tehnicheskie prilozhenija volnovyh processov, Ekaterinburg, №. 11, pp. 202-204 (2012)

6. Cameron R. J., Kudsia C. M., Mansour R. Microwave filters for communication systems. John Wiley \& Sons (2015)

7. R.E. Semernya et al., Design of compact bandpass quasi-elliptic microstrip filters, Journal of the Russian Universities, Radioelectronics, №. 6, pp. 41-53 (2019)

8. R.E. Semernya et al., Design of bandpass quasi-elliptic filter on cavity combine resonators for LTE frequency band, Radiotekhnika, Antenny, v. 44 № 10 (254), pp. 42-53 (2018) 